\title{
Intento suicida y factores asociados en dos instituciones de Cali - Colombia
}

\author{
Suicide attempts and associated factors in two institutions of Cali - Colombia
}

\author{
Tentativa de suicídio e fatores associados em duas instituições da Cali - Colômbia
}

\author{
Pedro Nel Beitia-Cardona* \\ Estela Rodríguez-Torres ${ }^{* *}$ \\ Catalina Estrada-González ${ }^{* * *}$ \\ Mercedes Benavides-Portilla ${ }^{* * * *}$
}

\section{Resumen}

Objetivo: Analizar prospectivamente el perfil epidemiológico y clínico de consultantes en servicios de urgencias por intento suicida, en dos instituciones de salud de Cali Colombia. Materiales y Métodos: Estudio observacional trasversal de consultantes en servicios de urgencias, sus historias clínicas y fichas de notificación; se analizan variables sociodemográficas, mecanismos, factores desencadenantes y de riesgo. Se realizó análisis descriptivo utilizando porcentajes e IC 95\%. Para el análisis bivariado se estimó OR simple e IC95\%. Los datos se procesaron en Epi info 7. Resultados: La edad media fue 28 años predominando mujeres con $63.81 \%$ (IC 95\% 56.91\% - 70.31\%), especialmente estudiantes en 29.05\% (IC 95\% 23.01\% $35.69 \%$ ). Depresión es la enfermedad mental presente con $43.33 \%$ (IC 95\% 36.53\% - 50.33\%). Ruptura sentimental con 36.19\% (IC 95\% 29.69\% - 43.09\%), es el factor desencadenante. Intoxicación con 70.95\% (IC 95\% 64.31\% - 76.99\%), el mecanismo más utilizado. $82.38 \%$ (IC 95\% 76.54\% - 87.28\%) lo intentaron por primera vez. Trastornos adaptativos, OR de 9.41 (IC 95\% 2.75-32.20), bajo rendimiento académico OR de 11.05 (IC 1.12\% 108.81), disfunción familiar OR de 3.15 (IC 95\% 1.66.20), consumo de benzodiacepinas OR de 4.62 (IC 95\% 1.34-15.90) y consumo de cocaína en hombres, OR de 5.5 (IC 95\% 1.68-17.94) fueron factores de riesgo. Conclusión: Es necesario implementar el sistema de vigilancia epidemiológica de conducta suicida acorde a la Ley 1616 de 2013, con base en factores desencadenantes y de riesgo identificados, brindar elementos para la formulación de políticas públicas encaminadas al mejoramiento de la convivencia social y la salud mental.

Palabras clave: Intento de suicidio, factores de riesgo, salud mental.

\section{Abstract}

Objective: Prospectively analyze the epidemiological and clinical profile of the consultants of emergency services for suicide attempt in two health institutions of Cali, Colombia. Materials and Methods: Cross-sectional observational study of consultants in emergency services, their clinical history and notification sheets; so-

\section{Autor de correspondencia}

* $\triangle$ Enfermero MSc. Epidemiología, Profesor Dedicación Exclusiva Facultad de salud, Departamento de Salud Pública. Universidad Santiago de Cali. Correo: pnbeitia@usc.edu. co Orcid: (iD https://orcid.org/0000-00028644-6008. Cali-Colombia.

** Profesora Dedicación Exclusiva Facultad de salud, Centro de Estudios e Investigación en Salud. Universidad Santiago de Cali. Correo: estelaroto@hotmail.com. Orcid: (iD) https://orcid.org/0000-0002-4911-627X. Cali-Colombia

*** Profesora Tiempo Completo Facultad de salud, Departamento de salud pública. Universidad Santiago de Cali. Correo: catalina. estrada00@usc.edu.co Orcid: iD https://orcid.org/0000-0002-8323-0973. Cali-Colombia

**** Profesora Dedicación Exclusiva Facultad de salud, Departamento de Salud Pública. Universidad Santiago de Cali. mbenavidez@ usc.edu.co Orcid: (iD https://orcid.org/00000001-7500-176X. 
ISSN-PRINT

1794-9831

E-ISSN 2322-7028

Vol. 16 No. 1

Ene - Abr 2019

Cúcuta, Colombia

ciodemographic variables, mechanisms, and trigger and risk factors are analyzed. A descriptive analysis was performed using percentages and CI 95\%. For the bivariate analysis simple OR and CI95\% was estimated. The data was processed in Epi info 7. Results: The median age was 28 years predominating women with $63.81 \%$ (CI 95\% 56.91\% - 70.31\%), especially students, 29.05\% (CI 95\% $23.01-35.69 \%$ ). Depression is the present mental illnesses with $43.33 \%$ (CI 95\% $36.53 \%$ - 50.33\%). Romantic breakup is the trigger factor with 36.19\% (CI 95\% 29.69\% - 43.09\%). Intoxication with 70.95\% (CI 95\% 64.31\% - 76.99\%), is the most used mechanism. 82.38\% (CI 95\% $76.54 \%-87.28 \%$ ) tried it for the first time. Risk factors were: adaptive disorder, OR of 9.41 (IC $95 \%$ 2.75-32.20), low academic performance OR of 11.05 (IC 1.12\% 108.81), family dysfunction OR of 3.15 (IC 95\% 1.6- 6.20), benzodiazepine consumption OR of 4.62 (CI 95\% 1.34-15.90) and male cocaine consumption, OR of 5.5 (CI 95\% 1.68-17.94). Conclusion: It is necessary to implement an epidemiological suicide conduct surveillance system according to Law 1616 of 2013, based on identified trigger and risk factors, provide elements for the formulation of public policies that aim for improvement of social coexistence and mental health.

Keywords: Suicide attempt, risk factors, mental health.

\section{Resumo}

Objetivo: Analisar prospectivamente o perfil epidemiológico e clínico de pacientes em serviços de emergência hospitalar por intento suicida, em dois instituições de saúde da cidade de Cali na Colômbia. Materiais e Métodos: Estudo observacional transversal de pacientes em serviços de emergências, suas histórias clínicas e cartões de notificação; se analisaram variáveis sócio-demográficas, mecanismos, fatores desencadeantes e de risco. Realizou-se análise descritiva utilizando porcentagens e IC 95\%. Para a análise bivariada se estimou OR simples e IC95\%. Os dados se processaram em Epi info 7. Resultados: A idade média foi 28 anos predominando mulheres com 63,81\% (IC 95\% 56,91\% - 70,31\%), especialmente estudantes em 29,05\% (IC 95\% 23,01\% - 35,69\%). Depressão é a doença mental presente com 43,33\% (IC 95\% 36,53\% - 50,33\%). Ruptura sentimental com $36,19 \%$ (IC 95\% 29,69\% - 43,09\%), é o fator desencadeante. Intoxicação com 70,95\% (IC 95\% $64,31 \%$ - 76,99\%), o mecanismo mais utilizado. 82,38\% (IC 95\% 76,54\% - 87,28\%) o intentaram por primeira vez. Transtornos adaptativos, OR de 9,41 (IC 95\% 2,75-32,20), baixo rendimento acadêmico OR de 11,05 (IC 1,12\% 108,81), disfunção familiar OR de 3,15 (IC 95\% 1,6- 6,20), consumo de benzodiazepinas OR de 4,62 (IC 95\% 1,34-15,90) e consumo de cocaína em homens, OR de 5,5 (IC 95\% 1,68-17,94) foram fatores de risco. Conclusão: é necessário implementar o sistema de vigilância epidemiológica de conduta suicida acorde à Lei 1616 de 2013, baseado em fatores desencadeantes e de risco identificados, fornecer os elementos para a formulação de políticas públicas encaminhadas ao melhoramento da convivência social e a saúde mental.

Palavras-chave: fatores de risco, intento de suicídio, saúde mental.

\section{Introducción}

El suicidio es un problema de gran relevancia, según la Organización Mundial de la Salud (OMS) para el año 2015 más de 800.000 personas se suicidan cada año en el mundo; el suicidio es la segunda causa de muerte en el grupo etario de 15-29 años (1). En el año 2020 duplicará su tasa, constituyéndose en una de las primeras causas de muerte a nivel mundial, generando un incremento en la carga social y económica de las poblaciones afectadas (2).

El intento suicida se refiere a los actos en los que la persona de forma intencional se provoca daño físico, pone en peligro su integridad y requiere de atención médica especializada para mitigar los daños producidos, así como un abordaje psiquiátrico oportuno para evaluar y manejar el trastorno mental subyacente (3). Las personas que intentan suicidarse esperan un resultado fatal, a pesar de que su intención sea ambigua, es decir, no ven a la muerte como el fin último.

Las cifras de suicidio a nivel mundial presentan un subregistro que alcanza el 50\%, esto hace que la mayoría de los países no muestren la magnitud real de este problema. Se ha podido estimar que por cada suicidio se registran de 10 a 25 intentos y de estos entre el 10 y el 15\% terminan suicidándose (4). 
En el intento suicida intervienen diversos factores desencadenantes como son los aspectos psicológi$\cos$, sociales, biológicos y éticos. La conducta suicida aumenta notoriamente a partir de las alteraciones psiquiátricas, en el $60 \%$ de los casos se evidencian problemas de depresión y en el $40 \%$ restante trastornos de personalidad, esquizofrenia, drogadicción y alcoholismo (5).

A pesar de esto no se pueden relacionar los trastornos psiquiátricos con el suicidio, pues no todos los pacientes con problemas mentales recurren a éste como solución a sus problemáticas. Esto evidencia que pueden existir otros factores que se relacionan con la vulnerabilidad y la impulsividad de la conducta suicida (5).

La Organización Mundial de la Salud (OMS) refiere que el suicidio es una prioridad de salud pública y se presenta anualmente en cerca de 800.000 personas; estima además que hay al menos 20 intentos fallidos por cada caso de muerte. Igualmente, señala que para el año 2015 fue la segunda causa de defunción en el grupo etario entre los 15 y 29 años en todo el mundo (6)

Entre los países con más altas tasas de suicidio se encuentran algunos del continente europeo, tales como: Hungría, Finlandia, Checoslovaquia, Austria, Alemania y Dinamarca, donde también ha ocurrido un aumento del intento suicida en niños y adolescentes (7).

En países de América, el suicidio figura en el tercer o cuarto lugar de las causas de defunciones en las edades comprendidas entre 15 y 44 años y representa 6,5 $\%$ del total de las muertes en este grupo etario. Según la OMS la tasa de suicidio se ha incrementado un 60 $\%$ en los últimos 45 años y actualmente se encuentra entre las principales causas de muerte en las personas de 15 a 44 años y de ambos sexos (8)

En Colombia el suicidio es considerado como un evento de baja magnitud en relación con el promedio mundial; sin embargo, para el año 2011 representó el $7 \%$ de las muertes violentas registrando una tasa de 4 por 100.000 habitantes (9).

La Ley 1616 de 2013 en el artículo 35 determina la implementación por parte del Ministerio de Salud y Protección Social el sistema de vigilancia de la conducta suicida; igualmente, en el plan Decenal de Salud Pública 2012-2021 establece como unos de sus componentes la promoción de la convivencia social y salud mental $(10,11)$.

Por tanto, al observar el intento de suicidio desde la epidemiología y la salud pública, se espera encontrar claramente identificados factores de riesgo comunes, perfiles sociales y demográficos bien caracterizados, $\mathrm{y}$ tendencias identificables.

La Encuesta Nacional de Salud Mental de 2003 (12), reveló que el $12,3 \%$ de los colombianos ha tenido ideaciones suicidas, el $4,1 \%$ ha realizado planes y el $4,9 \%$ ha hecho intentos suicidas.

En la Encuesta Nacional de Salud Mental del año 2015 (13), se reporta la prevalencia de intento de suicidio en las personas que han planeado hacerlo y que se encuentran en condiciones de pobreza que es del $40.3 \%$, mientras que en las personas que no se encuentran en esta condición es del $31.1 \%$. La ideación suicida en adolescentes de 12 a 17 años tiene una prevalencia de $6,6 \%$; el plan suicida tiene una prevalencia de $1,8 \%$; el intento suicida tiene una prevalencia de $2,5 \%$ y el $37,6 \%$ de los adolescentes que han pensado suicidarse lo han intentado.

De acuerdo con datos del Informe del Sistema de Vigilancia Epidemiológica de Cali 2014(14), en intento de suicidio se reportaron 248 casos; es decir, un aumento del $40 \%$ en número de casos en relación con los reportados en el 2013. El 66\% de los casos en el año 2014 ocurrieron en mujeres, lo que es similar a los años anteriores; sin embargo, es evidente el aumento del reporte en hombres, la relación de mujer: hombre para el año 2014 fue de 2:1. El 80\% de los que intentan suicidarse son menores de 35 años.

Con relación a los mecanismos para el intento suicida, Leal (15) hace referencia al uso de plaguicidas como el más utilizado para intentar quitarse la vida en un $48.4 \%$, situación que está asociada con la disponibilidad al interior de los hogares. Este mismo comportamiento fue identificado por Rodríguez et al. (16), quienes señalaron que en general las intoxicaciones fueron el principal mecanismo para el suicidio. Sin embargo, en los hombres el ahorcamiento fue mayor que las intoxicaciones, siendo diferencial por sexo, en las mujeres los porcentajes de estos dos mecanismos fue similar durante el periodo de estudio.

Por su parte Ceballos (17), plantea que entre los métodos más empleados para cometer suicidio están el 
ISSN-PRINT

1794-9831

E-ISSN 2322-7028

Vol. 16 No. 1

Ene - Abr 2019

Cúcuta, Colombia uso de arma de fuego, cortopunzantes y la intoxicación con sustancias venenosas. Llamando la atención sobre las diferencias por sexo, los hombres utilizan en un $64 \%$ arma de fuego frente a un $37 \%$ en las mujeres; en intoxicaciones, el 11\% de los eventos suicidas en los hombres ocurrieron por este mecanismo frente a un $40 \%$ de casos en mujeres.

Otros estudios encontraron resultados similares, siendo las intoxicaciones el primer o segundo mecanismo para este tipo de eventos $(18,19,20)$. Finalmente, en un estudio realizado por Cardona et al. (21), el 35.4\% de los eventos ocurrieron por ahorcamiento, seguido del uso de armas de fuego con un $26.6 \%$ e intoxicaciones, $29.7 \%$.

Conocer más en profundidad este fenómeno que incide sobre la salud pública de la población, que incrementa sustancialmente los años de vida potencialmente perdidos (AVPP), permitirá la implementación de medidas preventivas encaminadas a impactar esta problemática social que afecta en gran medida la dinámica poblacional en el mundo entero, especialmente en los países que se ven afectados por este evento.

\section{Objetivos}

\section{Objetivo General}

Analizar el perfil epidemiológico y clínico de consultantes en los servicios de urgencias por intento suicida en dos instituciones de salud de la ciudad de Cali Colombia.

\section{Objetivos Específicos}

- Caracterizar socio demográficamente las personas con intento de suicidio.

- Identificar los mecanismos utilizados para el intento de suicidio

- Determinar los factores de riesgo y desencadenantes del intento de suicidio.

\section{Materiales y Métodos}

Se realizó un estudio observacional de corte transversal a partir del total de consultantes a los servicios de urgencias, sus respectivas historias clínicas y las fichas de notificación de intento suicida (Código INS: 356) (22); se identificaron y analizaron 210 casos atendidos en dos Instituciones de Salud de Cali, una pública y otra privada, especializadas en el manejo del intento suicida.

La caracterización epidemiológica y clínica de los intentos suicidas se realizó con base a lo establecido en la ficha de notificación de intento suicida (Código INS: 356) (22) que permitió definir las variables de estudio, como factores desencadenantes constituidos por: problemas familiares, socioeconómicos, académicos y de salud; factores de riesgo identificando consumo de sustancias, ideación y/o pacto suicida, problemas familiares y trastornos psiquiátricos e intentos previos, al igual que los mecanismos utilizados.

La información se almacenó en una base de datos organizada en Excel antes de ser exportada a EpiI-Info 7. Para realizar el análisis univariado se calcularon frecuencias y porcentajes en el caso de las variables cualitativas; en las cuantitativas se calcularon medidas descriptivas. Para el análisis bivariado se calculó Odds Ratio simple (OR) como medida de asociación. En ella se empleó un nivel de significación de 95\%.

Se tuvo en cuenta las consideraciones éticas para el manejo de la información obtenida a partir de las fuentes de datos, como la confidencialidad y el anonimato definidas por la normatividad internacional y nacional $(23,24)$. Este estudio fue aprobado mediante Acta No. 01 de febrero 3 de 2013 del Comité de Ética y Bioética y aprobación por la Dirección General de Investigación de la Universidad DGI-COCEIN, No. 450-621013-010 del 15 de septiembre de 2013.

\section{Resultados}

Los hallazgos con respecto a la edad muestran la presencia del intento suicida entre los 12 y 78 años, con un promedio de 28 años. El 84.8\% de los intentos se concentraron entre los 10 y 39 años, siendo el grupo etario de 20 a 29 años con un 32.4\%, (IC 95\% 26.10\% - 39.16\%) el de mayor frecuencia, seguido de 10 y 19 con $30.00 \%$ (IC 95\% 23.89\% - 39.16\%). El mayor porcentaje de casos de intento suicida ocurrió en mujeres en un $63.8 \%$, (IC 95\% 56.91\% - 70.31\%). Las personas solteras fueron las que más lo intentaron en un $62.9 \%$, (IC 95\% 55.94\% - 69.41\%). Con relación a la ocupación u oficio, se identificó que los estudiantes en un $29.1 \%$ (IC 95\% 23.01\% - 35.69\%) intentaron suicidarse (Ver Tabla 1). 
Pedro Nel Beitia Cardona, Estela Rodríguez Torres, Catalina Estrada González, Mercedes Benavides

Tabla 1. Características Sociodemográficas de consultantes con intento suicida

\begin{tabular}{|c|c|c|c|c|c|}
\hline \multirow{2}{*}{\multicolumn{2}{|c|}{$\begin{array}{l}\text { VARIABLE } \\
\mathbf{n}=\mathbf{2 1 0}\end{array}$}} & \multirow{2}{*}{$\begin{array}{c}\text { MASCULINO } \\
\operatorname{Fr}(\%)\end{array}$} & \multirow{2}{*}{$\begin{array}{c}\text { FEMENINO } \\
\text { Fr/\%) }\end{array}$} & \multicolumn{2}{|c|}{ TOTAL } \\
\hline & & & & $\mathrm{Fr} / \%)$ & IC (95\%) \\
\hline \multirow{4}{*}{ Grupo de edad } & 10-19 años & $16(25.4)$ & $47(74.6)$ & $63(30.0)$ & $23.89-36.69$ \\
\hline & 20-29 años & $25(36.8)$ & $43(63.2)$ & $68(32.4)$ & $26.10-39.16$ \\
\hline & 30-39 años & $18(38.3)$ & $29(61.7)$ & $47(22.4)$ & $16.93-28.63$ \\
\hline & $40 \mathrm{y}+$ años & $17(53.1)$ & $15(46.9)$ & $32(15.2)$ & $10.66-20.83$ \\
\hline \multirow{5}{*}{ Estado civil } & Soltero & $44(33.3)$ & $88(66.7)$ & $132(62.9)$ & $55.94-69.41$ \\
\hline & Casado & $7(41.18)$ & $10(58.8)$ & $17(8.1)$ & $4.79-12.64$ \\
\hline & Unión Libre & $16(42.1)$ & $22(57.9)$ & $38(18.1)$ & $13.13-23.98$ \\
\hline & Separado & $8(40.0)$ & $12(60.0)$ & $20(9.5)$ & $5.91-14.33$ \\
\hline & Viudo & $1(33.3)$ & $2(66.7)$ & $3(1.4)$ & $0.30-4.12$ \\
\hline \multirow{8}{*}{ Ocupación } & Sin trabajo & $16(30.8)$ & $36(69.2)$ & $52(24.7)$ & $19.08-31.17$ \\
\hline & Estudiante & $16(26.2)$ & $45(73.8)$ & 61(29.1) & $23.11-35.69$ \\
\hline & Empleado & $20(52.6)$ & $18(47.4)$ & $38(18.1)$ & $13.13-23.98$ \\
\hline & Militar & $5(100.0)$ & - & $5(2.4)$ & $0.78-5.47$ \\
\hline & Hogar & - & $21(100.0)$ & $21(10.0)$ & $6.30-14.88$ \\
\hline & Pensionado & $2(100.0)$ & - & $2(1.0)$ & $0.12-3.40$ \\
\hline & Oficios varios & $14(56.0)$ & $11(44.0)$ & $25(11.9)$ & $7.85-17.07$ \\
\hline & Independiente & $3(50.0)$ & $3(50.0)$ & $6(2.9)$ & $1.06-6.11$ \\
\hline
\end{tabular}

Fuente: Base de Datos del estudio
En relación con la enfermedad mental al momento del intento suicida, la depresión fue la más frecuente en un $43.3 \%$ de los casos (IC 95\% 36.53\% - 50.33\%), seguida de la esquizofrenia con un 5,2\% (IC 95\% 2.64\% 9.18\%). De acuerdo con los trastornos mentales, la personalidad inestable en un $8.6 \%$ (IC 95\% 5.16\% - 13.21\%) y el trastorno de adaptación con un 6,2\% (IC 95\% 3.34\%
- 10,35\%), fueron los más evidentes (Ver Tabla 2).

El uso sustancias lícitas e ilícitas como el consumo de tabaco en un $14.3 \%$ (IC 95\% 9.85\% - 19.76\%), alcohol en un $13.8 \%$ (IC $95 \% 9.45 \%-19.23 \%$ ), marihuana en un $13.3 \%$ (IC 95\% 9.05\% - 18.69\%) y cocaína con un $7.1 \%$ (IC 95\% 4.05\% - 11.51\%) que son considerados factores de riesgo, estuvieron presentes (Ver Tabla 2). 
ISSN-PRINT

$1794-9831$

E-ISSN 2322-7028

Vol. 16 No. 1

Ene - Abr 2019

Cúcuta, Colombia

Tabla 2. Presencia de enfermedad, trastorno mental y consumo de sustancias licitas e ilícitas en el intento suicida

\begin{tabular}{|c|c|c|c|c|c|c|}
\hline & \multirow{2}{*}{$\begin{array}{c}\text { VARIABLE } \\
\mathrm{n}=210\end{array}$} & & \multirow{2}{*}{$\begin{array}{c}\text { MASCULINO } \\
\operatorname{Fr}(\%)\end{array}$} & \multirow{2}{*}{$\begin{array}{c}\text { FEMENINO } \\
\operatorname{Fr}(\%)\end{array}$} & \multicolumn{2}{|c|}{ TOTAL } \\
\hline & & & & & $\operatorname{Fr}(\%)$ & IC $(95 \%)$ \\
\hline \multirow{8}{*}{$\begin{array}{c}\text { Enfermedad } \\
\text { mental }\end{array}$} & \multirow{2}{*}{ Depresión } & SI & $30(33.0)$ & $2(67.0)$ & 91(43.3) & $36.53-50.33$ \\
\hline & & NO & $46(38.7)$ & $73(61.3)$ & $119(56.7)$ & $49.67-63.47$ \\
\hline & \multirow{2}{*}{ Esquizofrenia } & SI & $7(63.6)$ & $4(36.4)$ & $11(5.2)$ & $2.64-9.18$ \\
\hline & & $\mathrm{NO}$ & $69(34.7)$ & $130(65.3)$ & $199(94.8)$ & $90.82-97.36$ \\
\hline & \multirow{2}{*}{ Bipolaridad } & SI & $2(20.0)$ & $8(80.0)$ & $10(4.7)$ & $2.31-8.58$ \\
\hline & & $\mathrm{NO}$ & $74(37.0)$ & $126(63.0)$ & $200(95.2)$ & $91.42-93.69$ \\
\hline & \multirow{2}{*}{ Ansiedad } & SI & $1(25.0)$ & $3(75.0)$ & $4(1.9)$ & $0.52-4.80$ \\
\hline & & $\mathrm{NO}$ & $75(36.4)$ & 131(63.6) & $206(98.1)$ & $95.2-99.48$ \\
\hline \multirow{6}{*}{$\begin{array}{l}\text { Trastornos } \\
\text { mentales }\end{array}$} & \multirow{2}{*}{ Personalidad inestable } & SI & $6(33.3)$ & $12(66.7)$ & $18(8.6)$ & $5.16-13.21$ \\
\hline & & $\mathrm{NO}$ & $70(36.5)$ & $122(63.5)$ & 192(91.4) & $86.79-94.84$ \\
\hline & \multirow{2}{*}{ Trastorno de adaptación } & SI & $5(38.5)$ & $8(61.5)$ & $13(6.2)$ & $3.34-10.35$ \\
\hline & & $\mathrm{NO}$ & $71(36.0)$ & $126(64.0)$ & 197(93.8) & $89.65-96.66$ \\
\hline & \multirow{2}{*}{ Trastorno conversivo } & SI & $2(18.2)$ & $9(81.8)$ & $11(5.2)$ & $2.64-9.18$ \\
\hline & & $\mathrm{NO}$ & $74(37.2)$ & $125(62.8)$ & $119(94.8)$ & $90.82-97.36$ \\
\hline \multirow{12}{*}{$\begin{array}{l}\text { Consumo sus- } \\
\text { tancias lícitas } \\
\text { e ilícitas }\end{array}$} & \multirow{2}{*}{ Alcohol } & SI & $15(51.7)$ & $14(48.3)$ & $29(13.8)$ & $9.45-19.23$ \\
\hline & & $\mathrm{NO}$ & $61(33.7)$ & $120(66.3)$ & $181(86.2)$ & $80.77-90.55$ \\
\hline & \multirow{2}{*}{ Tabaco } & SI & $11(36.7)$ & $19(63.3)$ & $30(14.3)$ & $9.85-19.76$ \\
\hline & & $\mathrm{NO}$ & $65(36.11)$ & $115(63.9)$ & $180(85.7)$ & $80.24-90.15$ \\
\hline & \multirow{2}{*}{ Bazuco } & SI & $1(50.0)$ & $1(50.0)$ & $2(0.95)$ & $0.12-3.40$ \\
\hline & & $\mathrm{NO}$ & $75(36.1)$ & $133(63.9)$ & $208(99.1)$ & $96.60-99.88$ \\
\hline & \multirow{2}{*}{ Cocaína } & SI & $11(73.3)$ & $4(26.7)$ & $15(7.1)$ & $4.05-11.51$ \\
\hline & & $\mathrm{NO}$ & $65(33.3)$ & $130(66.7)$ & 195(92.9) & $88.49-45.95$ \\
\hline & \multirow{2}{*}{ Heroína } & SI & - & $1(0.8)$ & $1(0.48)$ & $0.01-2.62$ \\
\hline & & $\mathrm{NO}$ & $76(36.4)$ & 133(99.3) & $209(99.5)$ & $97.38-99.99$ \\
\hline & \multirow{2}{*}{ Marihuana } & SI & $11(39.3)$ & $17(60.7)$ & $28(13.3)$ & $9.05-18.69$ \\
\hline & & $\mathrm{NO}$ & $65(35.7)$ & $117(64.3)$ & $182(86.7)$ & $81.31-90.95$ \\
\hline
\end{tabular}

Fuente: Base de Datos del estudio

Entre los factores desencadenantes del intento suicida, la ruptura sentimental con $36.2 \%$ (IC 95\% $29.69 \%$ - 43.09\%) y la disfunción familiar con $29.1 \%$ (IC 95\% 23.01\% - 35.69\%) fueron las más frecuentes. La baja autoestima en $13.3 \%$ (IC 95\% 9.05\% $18.69 \%$ ) y los problemas financieros en $12.9 \%$ (IC $95 \% 8.65 \%$ - 18.15\%), se destacan en segundo orden de importancia (Ver Tabla 3).
El mecanismo más utilizado para el intento suicida fue la intoxicación en un 71.0\% (IC 95\% 64.31\% - 76.99\%), usándose principalmente benzodiacepinas en un 57.7\%, y los compuestos organofosforados en el $29.5 \%$. El uso de armas corto punzantes en el 20.0\% (IC 95\% 14.81\% $26.06 \%$ ) y el ahorcamiento en un 5.2\% (IC 95\% 2.64\% 9.18\%) fueron los menos frecuentes. El 82.4\% (IC 95\% $76.54 \%-87.28 \%$ ) realizaron un primer intento suicida, el resto presentaron intentos previos (Ver Tabla 3 ). 
Pedro Nel Beitia Cardona, Estela Rodríguez Torres, Catalina Estrada González, Mercedes Benavides Portilla

Tabla 3. Factores desencadenantes y mecanismos para el intento suicida

\begin{tabular}{|c|c|c|c|c|c|c|}
\hline & \multirow{2}{*}{$\begin{array}{l}\text { VARIABLE } \\
\qquad \mathbf{n}=\mathbf{2 1 0}\end{array}$} & & \multirow{2}{*}{$\begin{array}{c}\text { MASCULINO } \\
\operatorname{Fr}(\%)\end{array}$} & \multirow{2}{*}{$\begin{array}{c}\text { FEMENINO } \\
\operatorname{Fr}(\%)\end{array}$} & \multicolumn{2}{|c|}{ TOTAL } \\
\hline & & & & & $\operatorname{Fr}(\%)$ & IC (95\%) \\
\hline \multirow{12}{*}{$\begin{array}{c}\text { Factor } \\
\text { desencadenante }\end{array}$} & \multirow{2}{*}{ Ruptura sentimental } & SI & $22(29.0)$ & $54(71.1)$ & $76(36.2)$ & $29.69-43.09$ \\
\hline & & NO & $54(40.3)$ & $80(59.7)$ & $134(63.8)$ & $56.91-70.31$ \\
\hline & \multirow{2}{*}{ Baja autoestima } & SI & 11(39.3) & $17(60.7)$ & $28(13.3)$ & $9.05-18.69$ \\
\hline & & NO & $65(35.7)$ & $117(64.3)$ & $182(86.7)$ & $81.31-90.95$ \\
\hline & \multirow{2}{*}{ Disfunción familiar } & SI & $20(35.7)$ & $41(67.2)$ & $61(29.1)$ & $23.01-35.69$ \\
\hline & & NO & $56(37.6)$ & $93(62.4)$ & $149(71.0)$ & $64.31-76.99$ \\
\hline & \multirow{2}{*}{ Problema financiero } & SI & $12(44.4)$ & $15(55.6)$ & $27(12.9)$ & $8.65-18.15$ \\
\hline & & NO & $64(35.0)$ & $119(65.0)$ & $183(87.1)$ & $81.85-91.35$ \\
\hline & \multirow{2}{*}{ Desempleo } & SI & $4(23.5)$ & $13(76.5)$ & $17(8.1)$ & $4.79-12.64$ \\
\hline & & $\mathrm{NO}$ & $72(37.3)$ & $121(62.7)$ & 193(91.9) & $87.36-95.21$ \\
\hline & \multirow{2}{*}{ Intentos previos } & SI & $12(32.4)$ & $25(67.6)$ & $37(17.6)$ & $12.72-23.46$ \\
\hline & & NO & $64(34.0)$ & $109(63.0)$ & $173(82.4)$ & $76.54-87.28$ \\
\hline \multirow{8}{*}{ Mecanismos } & \multirow{2}{*}{ Arma corto punzante } & SI & $18(42.9)$ & $24(57.1)$ & $42(20.0)$ & $14.81-26.06$ \\
\hline & & NO & $58(34.5)$ & $110(65.5)$ & $168(80.0)$ & $73.94-85.19$ \\
\hline & \multirow{2}{*}{ Arma de fuego } & SI & $1(100.0)$ & - & $1(0.48)$ & $0.01-2.62$ \\
\hline & & NO & $75(35.9)$ & $134(64.1)$ & $209(99.5)$ & $97.38-99.99$ \\
\hline & \multirow{2}{*}{ Ahorcamiento } & SI & $5(45.5))$ & $6(54.6)$ & $11(5.2)$ & $2.64-9.18$ \\
\hline & & NO & 71(35.7) & $128(64.3)$ & 199(94.8) & $90.82-97.36$ \\
\hline & \multirow{2}{*}{ Intoxicación } & SI & $49(32.9)$ & $100(67.1)$ & $149(71.0)$ & $64.3-76.99$ \\
\hline & & NO & $27(44.3)$ & $34(55.7)$ & $61(30.0)$ & $23.01-35.69$ \\
\hline
\end{tabular}

$1794-9831$

E-ISSN 2322-7028

Vol. 16 No. 1

Ene - Abr 2019

Cúcuta, Colombia

Fuente: Base de Datos del estudio

Al comparar los consultantes menores de 18 años con los mayores de esta edad, se identificaron los siguientes factores de riesgo: los que presentan trastornos adaptativos en relación a los que no los presentaron OR de 9.41 (IC 95\% 2.75-32.20); bajo rendimiento académico frente a los que tuvieron un rendimiento académico adecuado OR de 11.05 (IC 95\% 1.12108.81), es de destacar que este fue un factor desen- cadenante al igual que la disfunción familiar OR de 3.15 (IC 95\% 1.6- 6.20). Por otro lado, el consumo de benzodiacepinas OR de 4.62 (IC 95\% 1.34-15.90) y los hombres que consumían cocaína en comparación con los que no lo hacían OR de 5.5 (IC 95\% 1.6817.94) presentaron asociación estadísticamente significativa (Ver Tabla 4). 
ISSN-PRINT

1794-9831

E-ISSN 2322-7028

Vol. 16 No. 1

Ene - Abr 2019

Cúcuta, Colombia

Tabla 4. Factores de Riesgo para el Intento de Suicidio para los menores de 18 años, Cali 2014-2015

\begin{tabular}{|c|c|c|c|c|c|c|}
\hline FACTOR & & $<18$ AÑOS & $>18$ AÑOS & OR & IC $(95 \%)$ & $\mathrm{p}$ \\
\hline \multirow{2}{*}{ Trastorno adaptativo } & SI & 9 & 38 & \multirow{2}{*}{9.41} & \multirow{2}{*}{$2.75-32.20$} & \multirow{2}{*}{0.000} \\
\hline & $\mathrm{NO}$ & 4 & 159 & & & \\
\hline \multirow{2}{*}{ Depresión } & SI & 15 & 32 & \multirow{2}{*}{0.54} & \multirow{2}{*}{$0.27-1.07$} & \multirow{2}{*}{0.072} \\
\hline & $\mathrm{NO}$ & 76 & 87 & & & \\
\hline \multirow{2}{*}{ Ansiedad } & SI & 1 & 46 & \multirow{2}{*}{1.16} & \multirow{2}{*}{$0.12-11.41$} & \multirow{2}{*}{0.89} \\
\hline & $\mathrm{NO}$ & 3 & 160 & & & \\
\hline \multirow{2}{*}{$\begin{array}{l}\text { Bajo rendimiento } \\
\text { académico }\end{array}$} & SI & 3 & 44 & \multirow{2}{*}{11.05} & \multirow{2}{*}{$1.12-108.81$} & \multirow{2}{*}{0.010} \\
\hline & $\mathrm{NO}$ & 1 & 162 & & & \\
\hline \multirow{2}{*}{ Baja autoestima } & SI & 5 & 42 & \multirow{2}{*}{0.72} & \multirow{2}{*}{$0.26-2.02$} & \multirow{2}{*}{0.54} \\
\hline & $\mathrm{NO}$ & 23 & 140 & & & \\
\hline \multirow{2}{*}{ Disfunción familiar } & SI & 23 & 24 & \multirow{2}{*}{3.15} & \multirow{2}{*}{$1.60-6.20$} & \multirow{2}{*}{0.000} \\
\hline & $\mathrm{NO}$ & 38 & 125 & & & \\
\hline \multirow{2}{*}{ Intentos previos } & SI & 8 & 39 & \multirow{2}{*}{0.95} & \multirow{2}{*}{$0.40-2.24$} & \multirow{2}{*}{0.90} \\
\hline & NO & 29 & 134 & & & \\
\hline \multirow{2}{*}{$\begin{array}{c}\text { Consumo benzodiace- } \\
\text { pinas }\end{array}$} & SI & 6 & 41 & \multirow{2}{*}{4.62} & \multirow{2}{*}{$1.34-15.90$} & \multirow{2}{*}{0.009} \\
\hline & $\mathrm{NO}$ & 5 & 158 & & & \\
\hline \multirow{2}{*}{ Consumo marihuana } & SI & 7 & 40 & 118 & $047 ?, 08$ & 077 \\
\hline & $\mathrm{NO}$ & 21 & 142 & 1.10 & $0.41-2.90$ & 0.12 \\
\hline Concump cocoín & SI & 11 & 65 & 55 & $160-1704$ & 0005 \\
\hline 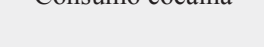 & $\mathrm{NO}$ & 4 & 130 & 0.0 & דל דות & 0.000 \\
\hline Concume alcohol & SI & 3 & 44 & 036 & 01014 & 000 \\
\hline coirsuinto aic volivo & $\mathrm{NO}$ & 26 & 137 & 0.00 & $0.10-1.24$ & 0.03 \\
\hline Intovinocí́ & SI & 35 & 12 & 125 & $060 ?$ & 0.55 \\
\hline 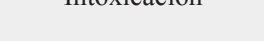 & $\mathrm{NO}$ & 114 & 49 & 1.20 & $0.00-2.02$ & 0.00 \\
\hline
\end{tabular}

Fuente: Base de Datos del estudio

\section{Discusión}

El intento de suicidio se considera un problema de salud pública que afecta a la población en general, especialmente a los adolescentes, en esta etapa del ciclo vital se presenta mayor vulnerabilidad por cuanto se hacen más evidentes las exigencias psicosociales, las relaciones familiares, académicas y laborales (25).

Con respecto a la edad de intento de suicidio, los resultados obtenidos son similares a los encontrados por Cañón Buitrago et al. (26) quienes reportaron un $33.3 \%$ en el grupo de 20 a 29 años. Sin embargo, se observa una diferencia con respecto a lo obtenido por Valencia Upegui et al., (27) quienes encontraron un incremento significativo en el grupo de 15 a 24 años, pasando de $4.9 \%$ a $37.7 \%$.
La encuesta Nacional de Salud Mental del 2015 (13), muestra que la prevalencia de intento suicida en personas de 12 a 17 años es de $2.5 \%$ y el $37.6 \%$ de estos individuos han intentado suicidarse. En el estudio se identificó que el 30\% de las personas que intentaron suicidarse, estaban entre los 10 y 19 años. Este cambio puede estar relacionado con los factores de riesgo identificados como los trastornos adaptativos y la disfunción familiar que se presentan con más frecuencia en este grupo de edad; de ahí que sea necesario focalizar las estrategias de prevención en las personas de este grupo etario.

En cuanto al género, el intento de suicidio es más frecuente en el femenino, resultados similares fueron encontrados en estudios realizados por: Pérez Collado et al., (25) (88.0\%), Cañón Buitrago et al., (26) 
(67\%) y Valencia Upegui (27) (56.4\%). Esto supone que los hombres son más efectivos que las mujeres al momento de querer quitarse la vida debido a que ellos utilizan métodos más violentos y letales.

En cuanto al estado civil, los solteros fueron los que más lo intentaron, resultado semejante con estudios realizados por Valencia Upegui (27) (63.6\%) y Cañón Buitrago et al (25) (54\%). La condición de vivir solo o no tener una pareja estable puede ser un factor que predispone a querer quitarse la vida, esto no implica que no se deban de tener en cuenta otras condiciones de convivencia como el estar casado o tener una relación de pareja estable.

De acuerdo con la ocupación, se identificó que los estudiantes son los que más intentan suicidarse, dato que coincide con el estudio de Valencia Upegui (27), quien encontró que el $26.3 \%$ de los que lo intentan, eran estudiantes.

El ambiente académico es considerado generador de altos niveles de estrés relacionados con la responsabilidad que tienen los estudiantes con su formación y su salud, enfrentándose a una gran cantidad de responsabilidades cognitivas, las cuales prolongándose en el tiempo pueden afectar la salud mental de esta población, lo que puede relacionarse con el Bajo Rendimiento Académico y las rupturas sentimentales que son muy frecuentes en la población que desempeña esta ocupación (28).

Con relación a la enfermedad mental presente al momento del intento, la depresión fue el más frecuente, resultado similar al reportado en el estudio de Valencia Upegui (27) donde se presentó en el $45.1 \%$ y el realizado por Cañón Buitrago et al., (26) donde se halló en el $33.3 \%$ de los casos estudiados.

La depresión es una de las enfermedades mentales de más alta prevalencia en la población y al inicio de los cuadros depresivos suelen tener en ocasiones síntomas que pasan desapercibidos para la persona y su entorno. Es por eso que el manejo médico no se hace de manera oportuna, solo hasta que se presente un desencadenante que ocasione la crisis o gestos de autoagresión como pueden ser las rupturas sentimentales, la disfunción familiar y el bajo rendimiento académico, entre otros. (6)

Respecto a los trastornos mentales, el de personalidad inestable fue más frecuente; en un estudio realizado por Coronado Molina (29) en Perú arrojó que el 25\% de los casos estudiados presentaron este tipo de trastorno. De igual forma, se considera que la poca tolerancia a la frustración, que está relacionada con este tipo de trastorno, conlleva a que las personas realicen actos impulsivos sin medir las consecuencias y en ocasiones incluso intentar quitarse la vida.

Entre los factores desencadenantes del intento suicida, la ruptura sentimental fue uno de los más frecuentes, resultados que fueron reportados de manera similar en el estudio realizado por Coronado Molina (29), donde la ruptura de la relación y el haber tenido una discusión previa fueron las situaciones de pareja más asociadas a las crisis, ambas en un $26 \%$ de los casos. Por otro lado, el estudio de Cañón-Buitrago et al., (26) evidenció que en el $66.7 \%$ de los casos la ruptura sentimental era desencadenante. El estudio de Pérez-Olmos y et al., (30), demostró que un 26\% de los casos, se relaciona con la ruptura sentimental como factor precipitante del intento de suicidio.

La disfunción familiar fue la segunda causa identificada como factor desencadenante de intento suicida. Resultado que es similar al reportado por Cañón Buitrago et al., (26) con un 37.5\%, Valencia Upegui (27) en $16 \%$ y Coronado Molina (31) en un $15 \%$. "Trabajar la integralidad de la familia resaltando, apoyo, comprensión, respeto y amor incondicional son aspectos fundamentales que las familias deben luchar cotidianamente por mantener y conservar" como lo plantean Olaya Cuadros y Gaviria Noreña (32).

Los datos anteriores se constituyen en un aspecto fundamental para el trabajo en familias donde se evidencie la disfunción familiar y así realizar acciones preventivas e impactar sobre este aspecto relacionado con el intento suicida.

La intoxicación fue el mecanismo utilizado con más frecuencia, destacándose el uso de benzodiacepinas y compuestos organofosforados. Valencia Upegui (27), encontró el consumo de sustancias pesticidas como el de mayor incidencia, con el 33,5\%, seguido por el uso de sustancias medicamentosas con 29,3\%. Por otro lado, Coronado-Molina (31) reportó el uso de carbamatos en el $54 \%$ y el uso de psicofármacos en un 38\%. La utilización de este tipo de mecanismo puede estar relacionado con la facilidad en su obtención, bien sea en el lugar de residencia o en el sitio donde intentan hacerlo y como se trata de intentos suicidas los mecanismos utilizados suelen ser de menor letalidad. 
ISSN-PRINT

1794-9831

E-ISSN 2322-7028

Vol. 16 No. 1

Ene - Abr 2019

Cúcuta, Colombia
La mayoría de las personas intentaron suicidarse por primera vez, resultados que se asemejan a los encontrados por Cañón Buitrago et al., (26) y Valencia Upegui (27) quienes reportan el $63.3 \%$ y $70.1 \%$ respectivamente. Esto evidencia que cada vez las personas intentan quitarse la vida como medio de resolución de conflictos, esto sugiere que debe brindase mayor atención y seguimiento a las personas mediante redes de apoyo utilizando diferentes técnicas grupales e individuales, porque se ha demostrado que los que lo intentan por primera vez lo seguirán intentando hasta lograr su cometido; técnicas que permitirán efectuar la adecuada prevención y manejo.

Con respecto a los factores de riesgo, se encontró que, en los menores de 18 años, la disfunción familiar fue uno de ellos, dato que es coincidente con lo reportado por el estudio de Pérez Olmos et al. (30) (OR 3.4 IC $95 \%$ 1.2-9.7). Gaona Ordoñez (31) et al., reportan que existe una asociación estadísticamente significativa entre la disfunción familiar y el intento de suicidio. Otros de los factores encontrados en el presente estudio fueron los trastornos adaptativos asociados a depresión. Olmos Pérez et al. (30) reportan la depresión como un factor de riesgo suicida (OR 27.9 IC 95\% 3.5-223.1).

El consumo de cocaína especialmente en los hombres también se comportó como factor de riesgo. González Forteza et al., (33) encontraron asociación significativa entre el intento de suicidio y el consumo de cocaína y otras sustancias alucinógenas; además, lo relacionan con el aumento de la frecuencia del intento. Igualmente, Fructuoso-Castellar (34) encontraron asociación entre el consumo de cocaína y el intento suicida (OR de 3.7 IC 95\% 1.6-8.4).

El bajo rendimiento académico en los menores de 18 años también se comportó como factor de riesgo, resultado que coincide con lo encontrado por Pinzón Amado et al. (35), quienes reportan que existe una asociación estadísticamente significativa entre el bajo rendimiento académico y el intento de suicidio (OR: 2,2; IC 95\%: 1,38-3,63).

El uso de Benzodiacepinas en el presente estudio se relaciona como factor de riesgo. Courtney, Bagge, y Borges (36) encontraron relación entre el consumo de ansiolíticos y opioides como factor de intento de suicidio $(\mathrm{OR}=2,82$; IC del 95\%, 1,13-7,01); sin embargo, Fructuoso-Castellar (34) no lo encontraron como factor de riesgo, pero sugieren que el consumo de es- tas sustancias en dosis muy elevada puede ser letal y de hecho algunas de estas intoxicaciones accidentales y como intento de suicidio pueden llegar a ser mortales, esto también está asociado a la facilidad con la que se puede acceder a este tipo de medicamentos por parte de las personas que intentan suicidarse.

Es pertinente destacar un número importante de casos que no fueron reportados con la respectiva ficha de notificación obligatoria según lo establecido en el protocolo de vigilancia en salud pública sobre el intento de suicidio, lo que sigue generando un subregistro de este evento, hecho que limitó al grupo investigador obtener más información y mayor conocimiento del comportamiento del intento suicida en la población.

Es necesario que las instituciones de salud hagan uso de los documentos diseñados para notificar y registrar este evento y de esta manera, tener una mejor evidencia del comportamiento de esta contingencia y que no sea tomada sólo como enfermedad mental al momento de la consulta, pasando por alto los factores desencadenantes que son de vital importancia en la intervención con fines de prevención del intento suicida y así evitar las recurrencias que cada vez son más frecuentes.

\section{Conclusiones}

- Se puede evidenciar que el fenómeno del intento suicida tiene un comportamiento de género, siendo las mujeres jóvenes las que más acuden a esta práctica como método de resolución de problemas de pareja; en algunos casos también están los relacionados con vacíos afectivos que provocan la disfunción familiar, los cuales actúan también como factores de riesgo.

- Dado que se trata de intento suicida, los mecanismos para hacerlo fueron menos letales, por lo cual la intoxicación especialmente con medicamentos fue la más utilizada.

- Se destacan además como factores de riesgo, el bajo rendimiento académico y los trastornos adaptativos, aspectos que hoy día son muy frecuentes en la población. Es por eso, que las personas que los sufren, ante una situación difícil en la cotidianidad opten por quitarse la vida.

- El consumo de cocaína y benzodiacepinas fueron 
otros factores de riesgo identificados en la población masculina, debido a la facilidad que se tiene hoy en día para acceder a ellos; razón por la cual se han constituido en mecanismos muy utilizados especialmente por los jóvenes para intentar suicidarse.

- Se hace entonces necesario implementar el sistema de vigilancia epidemiológica de la conducta suicida acorde a lo establecido en la Ley 1616 de 2013 y con base en los factores desencadenantes y de riesgo definidos en la ficha de noti- ficación y que fueron identificados en el estudio. Igualmente, se deben brindar elementos para la formulación de políticas públicas encaminadas al mejoramiento de la convivencia social y la salud mental en esta población, implementando redes de apoyo que permitan un abordaje integral y con base en ello realizar una adecuada prevención.

\section{Conflicto de intereses}

Los autores declaran no tener ningún conflicto de interés.

\section{Referencias Bibliográficas}

1. Cañón Buitrago SC, Castaño Castrillón JJ, Muriel Arriguí DL, et al. Factor de riesgo suicida y factores asociados en reclusas de un centro penitenciario de Caldas-Colombia. Arch Med [Internet] 2015. [Consultado 08 febrero de 2017]; 16(1):61-73. Disponible en: http://www.redalyc.org/articulo. oa?id=273846452007

2. Vargas Sanabria M, Vega Chaves JC, Montero Solano G, et al. Suicidio en adultos mayores en Costa Rica durante el periodo 2010-2014. Med. leg. Costa Rica [Internet] 2017. [Consultado 08 febrero de 2017]; 34(1): 2-25 Disponible en: http://www.scielo.sa.cr/scielo.php?script=sci_arttext\&pid $=\mathrm{S} 1409-00152017000100002$

3. Bando D, Brunoni A, Fernandes T, Benseñor I, Lotufo P. Suicide rates and trends in Sao Paulo, Brazil, according to gender, age and demographic aspects: a join-point regression analysis. Rev. Bras Psiquiatr [Internet] 2012. [Consultado 10 marzo de 2017]; 34: 268-293. Disponible en: http://www. scielo.br/scielo.php?script $=$ sci_arttext\&pid=S1516-44462012000300008

4. OMS. Suicidio. Centro de Prensa, Nota descriptiva. [Internet]. 2018 [Consultado 15 de abril de 2018] Disponible en http://www.who.int/mediacentre/factsheets/fs398/es/

5. Baca-García E, Aroca F. Factores de riesgo de la conducta suicida asociados a trastornos depresivos y ansiedad. Rev. Salud Mental [Internet].2014; [Consultado 15 de noviembre de 2017]; 37(5): 373-380. Disponible en http://www.scielo.org.mx/scielo.php?script=sci_arttext\&pid=s0185-33252014000500003

6. OMS. Informe sobre Suicidios: Cifras, Causas y Países que más lo padecen [Internet] 2018; [Consultado 27 de junio de 2018] Disponible en: www.who.int/es/news-room/fact-sheets/detail/suicide

7. Sánchez Sosa JC, Villarreal González ME, Musitu G, Martínez Ferrer B. Ideación Suicida en Adolescentes: Un Análisis Psicosocial. Psychosocial Intervention [Internet]. 2010; [Consultado junio 27 de 2018]; 19 (3):279-287. Disponible en: http:/www.redalyc.org/pdf/1798/179817507008.pdf

8. CortésAlfaro A. Conducta suicida adolescencia y riesgo. Rev. Cubana de Medicina General Integral [Internet]. 2013; [Consultado 27 de junio de 2018] 30 (1): 132-139. Disponible en http://www.scielo. sld.cu/pdf/mgi/v30n1/mgi13114.pdf

9. Macana Tuta NL. Comportamiento del suicidio en Colombia. Med Legal. Colombia [Internet] 2011. [Consultado 8 de febrero de 2017] Disponible en: http://www.medicinalegal.gov.co/ documents/10180/34616/6-F-11-Suicidio.pdf/6b2966e7-cbcb-4618-a3c3-af5cd111629e

10. INS. Protocolo de Vigilancia en Salud Pública. Intento de suicidio [Internet]. 2017 [Consultado 10 de febrero de 2018] Disponible en: https:/www.minsalud.gov.co/sites/rid/Lists/BibliotecaDigital/ RIDE/IA/INS/protocolo-vigilancia-intento-suicidio.pdf

11. Congreso de la República. Ley 1616 de 2013, enero 21, por medio de la cual se expide la Ley de salud mental y se dictan otras disposiciones. [Internet] Bogotá, D. C: Gobierno Nacional; 2013; [Consultado 
ISSN-PRINT

1794-9831

E-ISSN 2322-7028

Vol. 16 No. 1

Ene - Abr 2019

Cúcuta, Colombia
8 de febrero de 2017]. Disponible en https://www.minsalud.gov.co/sites/rid/Lists/BibliotecaDigital/ RIDE/DE/DIJ/ley-1616-del-21-de-enero-2013.pdf

12. Medina Pérez O, Cardona Duque DV, Arcila López SC. Riesgo suicida y depresión en un grupo de internos de una cárcel del Quindío (Colombia). Rev. Investigaciones Andina [Internet].2011; [Consultado 8 de febrero de 2017]; 13 (23):268-280. Disponible en: http://www.redalyc.org/articulo. oa? id $=239019089004$

13. Ministerio de salud y Protección social. Encuesta Nacional de Salud Mental 2015. Disponible en: https:/www.minsalud.gov.co/sites/rid/Lists/BibliotecaDigital/RIDE/DE/presentacion-encuesta-nacional-salud-mental-2015.pdf

14. Secretaría de Salud Pública Santiago de Cali. Boletín No. 8 Intento de Suicidio. Cali 2006 - 2014 [Internet] 2014. [Consultado noviembre 15 de 2017] Disponible. www.cali.gov.co/salud/publicaciones/ descargar.php?id $=42047$

15. Leal Zaval R. Caracterización del suicidio consumado en la división médico legal de Cajamarca y Cajabamba. Rev. Perspectiva. [Internet] 2016; [Consultado 15 de noviembre de 2017]; 17 (1): 77-92. Disponible en: http://revistas.upagu.edu.pe/index.php/PE/article/view/505/429

16. Rodríguez JA, Medina OA, Cardona DV. Caracterización del Suicidio en el departamento de Risaralda Colombia, 2015-2010. Rev. Fac. Med. [Internet] 2013; [Consultado 16 de noviembre de 2017]; 61: 9-16. Disponible en: https://revistas.unal.edu.co/index.php/revfacmed/article/view/39619/47324

17. Ceballos Ospino GA. El suicidio en Colombia durante el año 2015. Duazary Rev. Internacional de Ciencias de la Salud. [Internet] 2017; [Consultado 16 de noviembre de 2017]; 14 (1): 6-7. Disponible en: DOI: http://dx.doi.org/10.21676/2389783X.1812

18. Instituto Nacional de Medicina Legaly Ciencias Forenses. Suicidioen Colombia[Internet] 2006. [Consultado 25 de agosto 2016] Disponible en: http://www.medicinalegal.gov.co/documents/88730/1656998/ Forensis+Interactivo+2014.24-JULpdf.pdf/9085ad79-d2a9-4c0d-a17b-f845ab96534b

19. Franco SA, Gutiérrez ML, Sarmiento J, Cuspoca D, Tatis J, Castillejo, A, Barrios, M, BallesterosCabrera, MdP Zamora,S,Rodríguez, Cl. Suicidio en estudiantes universitarios en Bogotá, Colombia, 2004-2014. Ciência \& Saúde Coletiva [Internet] 2017; [Consultado 16 de noviembre de 2017] 22 (1): 269-278. Disponible en: http://www.redalyc.org/pdf/630/63049169031.pdf

20. Calderón Ramírez V, Alcocer Olaciregui A, Vargas Moranth R. Intento de suicidio por intoxicación con sustancias químicas en Colombia 2007-2013. Rev. Internacional de Ciencias de la Salud Duazary. [Internet] 2017; [Consultado 16 de noviembre de 2017]; 14 (2): 149-159. Disponible en: http://revistas.unimagdalena.edu.co/index.php/duazary/article/view/1963/1423

21. Cardona Arango D, Medina Pérez OA, Cardona Duque DV. Caracterización del Suicidio en Colombia 2000-2010. Rev.colomb.psiquiatr. [Internet] 2016; [Consultado 16 de noviembre de 2017];45 (3): 170-177 Disponible en: http://www.elsevier.es/es-revista-revista-colombiana-psiquiatria-379-articulo-caracterizacion-del-suicidio-encolombia-2000-2010-S0034745015001584

22. SIVIGILA. Subsistema de información SIVIGILA Ficha de notificación. Intento de suicidio. Código INS: 356. [Internet] 2017; [Consultado 16 de noviembre de 2017]. Disponible en http://www.clinicamedihelp.com/documentos/fichas/Intento\%20de\%20suicidio\%20356.pdf

23. Declaración Helsinki de la AMM [Internet] 2017; [Consultado 9 de febrero de 2017]. Disponible en: https://www.wma.net/es/policies-post/declaracion-de-helsinki-de-la-amm-principios-eticos-paralas-investigaciones-medicas-en-seres-humanos/

24. Ferro M, Molina L, Rodríguez W. La bioética y sus principios. Rev. Acta Odontológica Venezolana [Internet] 2009; [Consultado 15 de noviembre de 2016]; 47(2) Disponible en: http://www.actaodontologica.com/ediciones/2009/2/art26.asp

25. Pérez Collado J, Pérez Collado T, Azcuy Collado M, Mirabal Martínez G. Intento suicida en adolescentes un problema de salud en la comunidad. Rev. Cubana de investigaciones biomédicas [Internet] 
2014; [Consultado 5 de abril de 2017]; 33(1): 70-80. Disponible en: http://scielo.sld.cu/pdf/ibi/v33n1/ ibi08114.pdf

26. Cañón Buitrago S, Foro Duque P. Caracterización de la población con conducta suicida en la clínica San Juan de Dios de Manizales de Junio a noviembre de 2010. Rev. Universidad de Manizales [Internet] 2012; [Consultado 5 de abril de 2017] 12 (1): 83-92. Disponible en: http://www.redalyc.org/ pdf/2738/Resumenes/Resumen_273824148008_1.pdf

27. Valencia Upegui H, Campo Cabal G, Borrero Cortés C, García Gallardo AM, Patiño García MM. Caracterización de la población con intento de suicidio en el Hospital Universitario del Valle, Cali (1994-2010). Rev. Colombiana de Psiquiatría [Internet] 2011; [Consultado 20 de enero de 2017] 40 (4): 619-636. Disponible en http://www.redalyc.org/articulo.oa?id=80622345002

28. Mogollón Canal OM, Villamizar Carrillo DJ, Padilla Sarmiento SL. Salud mental en la educación superior: una mirada desde la salud pública. Rev. cienc. cuidad. [Internet] 2016; [Consultado 17 de mayo de 2017]; 13(1): 103-120. Disponible en: http://revistas.ufps.edu.co/ojs/index.php/cienciaycuidado/ article/viewFile/738/716

29. Coronado Molina $C$, Características clínico - epidemiológicas y abordaje terapéutico de pacientes que acuden por intento suicida al Hospital General de San José Callao, Perú. Rev. Neuropsiquiatría [Internet] 2014; [Consultado 17 de mayo de 2017]; 77 (4). Disponible en: http://www.scielo.org.pe/pdf/rnp/ v77n4/a07v77n4.pdf

30. Olmos Pérez I, Téllez Cruz DL, Vélez Traslaviña AL, Ibáñez Pinilla M. Caracterización de factores asociados con comportamiento suicida en adolescentes estudiantes de octavo grado en tres colegios bogotanos. Rev. Colombiana de Psiquiatría [Internet] 2012; [Consultado 17 de mayo de 2017]; 41 (1): 28-47. Disponible en: http://www.redalyc.org/pdf/806/80624093004.pdf

31. Gaona Ordoñez JB, Bohórquez de Figueroa A, Gómez Gómez PA, et al. Incidencia de los antecedentes de funcionalidad familiar, consumo de sustancias psicoactivas y enfermedad mental frente al intento de suicidio. Rev. cienc. Cuidad [Internet] 2012; [Consultado 17 de noviembre de 2017]; 9(2) :36-42. Disponible en: http://revistas.ufps.edu.co/ojs/index.php/cienciaycuidado/article/view/337/358

32. Olaya Cuadros BL, Gaviria Noreña DL. Cuidado de la salud de la familia. Rev. cienc. cuidad. [Internet] 2017; [Consultado 15 de agosto de 2017]; 14(1): 79-94. Disponible en: http://revistas.ufps.edu.co/ ojs/index.php/cienciaycuidado/article/view/808/951

33. González Forteza C, Juárez López CE, Montejo León LÁ, et al. Ideación suicida y su asociación con drogas, depresión e impulsividad en una muestra representativa de estudiantes de secundaria del estado de Campeche, México. Rev.Acta Universitaria [Internet] 2015; [Consultado 8 de Febrero de 2017]; 25 (2) : 29-34. Disponible en http://www.redalyc.org/pdf/416/41648310005.pdf

34. Fructuoso Castellar A. Medicamentos y Conducta Suicida. Un estudio de Casos y Controles. [Tesis Doctoral]. Universidad de Valladolid, Facultad de Medicina. 2016. Disponible en: http://uvadoc.uva. es/handle/10324/16382

35. Pinzón Amado A, Guerrero S, Moreno K, Landínez C, Pinzón J. Ideación suicida en estudiantes de medicina: prevalencia y factores asociados. Rev.colomb.psiquiatr [Internet] 2013; [Consultado 16 de noviembre de 2017]; 42 (1): 47-55. Disponible en: http://www.scielo.org.co/scielo.php?script=sci_ar ttext\&pid=S0034-74502013000500007

36. Courtney L, Bagge, and Borges G. Acute Substance Use as a Warning Sign for Suicide Attempts: A Case-Crossover Examination of the 48 Hours Prior to a Recent Suicide Attempt. J Clin Psychiatry [Internet] 2017; [Consultado 16 de noviembre de 2017]; 78(6):691-696. Disponible en: http://www. psychiatrist.com/JCP/article/Pages/2017/v78n06/v78n0610.aspx 